\title{
Continuum dynamics of the intention field under weakly cohesive social interaction
}

\author{
Pierre Degond \\ Department of Mathematics, Imperial College London, \\ South Kensington Campus, London SW7 $2 A Z$, UK \\ pdegond@imperial.ac.uk \\ Jian-Guo Liu \\ Department of Physics, Duke University, \\ Durham, NC 27708, USA \\ Department of Mathematics, Duke University, \\ Durham, NC 27r08, USA \\ jliu@phy.duke.edu \\ Sara Merino-Aceituno \\ Department of Mathematics, Imperial College London, \\ South Kensington Campus, London SW7 2AZ, UK \\ s.merino-aceituno@imperial.ac.uk \\ Thomas Tardiveau \\ École Polytechnique, Route de Saclay, \\ Paris, 91128 Palaiseau, France \\ thomas.tardiveau@polytechnique.edu
}

Received 20 July 2016

Accepted 6 August 2016

Published 9 January 2016

Communicated by N. Bellomo, F. Brezzi and M. Pulvirenti

\begin{abstract}
We investigate the long-time dynamics of an opinion formation model inspired by a work by Borghesi, Bouchaud and Jensen. First, we derive a Fokker-Planck-type equation under the assumption that interactions between individuals produce little consensus of opinion (grazing collision approximation). Second, we study conditions under which the Fokker-Planck equation has non-trivial equilibria and derive the macroscopic limit (corresponding to the long-time dynamics and spatially localized interactions) for the evolution of the mean opinion. Finally, we compare two different types of interaction rates: the original one given in the work of Borghesi, Bouchaud and Jensen (symmetric binary interactions) and one inspired from works by Motsch and Tadmor (non-symmetric binary interactions). We show that the first case leads to a conservative model for the density
\end{abstract}

This is an open access article published by World Scientific Publishing and distributed under the terms of the Creative Commons Attribution (CC BY) 4.0 License, which permits use, distribution and reproduction in any medium, provided the original author(s) and source are credited. 
of the mean opinion whereas the second case leads to a non-conservative equation. We also show that the speed at which consensus is reached asymptotically for these two rates has fairly different density dependence.

Keywords: Opinion formation; grazing limit; non-symmetric rate; continuum limit; Deffuant-Weisbuch model.

AMS Subject Classification: 82C21, 82C22, 82C26, 82C31, 82C40, 82C70, 91B12, 91B14, 91B70, 91B72

\section{Introduction}

The goal of this paper is the investigation of an opinion formation model inspired from the one presented in Ref. 10. First, we obtain the mean-field equations for this model and approximate the dynamics under the assumption that interactions between individuals produce little convergence of opinions (weak consensus interaction). We study the equilibria for this case and show that, under some conditions, it corresponds to a Gaussian distribution $\mathcal{N}\left(\varphi, \sigma^{2}\right)$ with a fixed given variance $\sigma^{2}$ but undetermined mean $\varphi$. The final aim is to derive the equation for the evolution of the mean opinion $\varphi$ in the spatially heterogeneous case when interactions become localized. During this analysis, we will consider two different cases corresponding to two different types of interaction rates: the original one given in Ref. 10 and one inspired from Refs. 29 and 30. We show that, asymptotically, the dynamics for the second rate reaches consensus faster in regions of low density of individuals while for high density regions, the dynamics corresponding to the first rate is faster in reaching consensus. The main result is discussed in the next section. As far as we know, this is the first result that derives the macroscopic dynamics for these equations.

The tools used to carry out the present analysis are borrowed from kinetic theory and hydrodynamic limit techniques which originally were developed to tackle problems from mathematical physics. Recently these tools have found applications in the study of emergent phenomena in biological and social systems. Some illustrative examples of this are the study of self-organized collective behavior in different settings like swarming and flocking, ${ }^{19,28}$ fish schools,,${ }^{1,11}$ ant trail formation ${ }^{20}$ or collective cell migration ${ }^{37}$; evolution of traffic ${ }^{26}$ and crowd dynamic ${ }^{3,27}$; the emergence of languages, ${ }^{36}$ cultures, $^{2}$ segregation ${ }^{31}$ or social classes. ${ }^{6}$ In particular, recently new approaches have been introduced to describe the formation of opinions using mean-field (or kinetic) equations. ${ }^{4,21,32,34}$

However, the modeling of opinion formation has a long history. One can trace it back to the Condorcet method for voting systems (1785) and more recently to the Fisher-KPP equation (1937) which has found applications in modeling rumor spreading. ${ }^{7}$ This was followed in 1971 by Ref. 35 that models polarization phenomena in a society, and in 1982 with the emergence of the concept of sociophysics. ${ }^{24}$ One of the first approaches to opinion formation consisted of describing society as a graph, or network, with individuals located at nodes and interacting with their neighboring nodes. Such a description fostered links with the Ising model where 
spins were replaced by an opinion variable. This first approach also encouraged to view opinions as discrete-valued variables. The main difference between the proposed models is the rule by which opinions evolve. The interested reader can find examples in the voter's model, ${ }^{12}$ the majority rule model ${ }^{23}$ and the Sznajd model. ${ }^{33}$ In opposition to this, in the model treated here opinions are represented by continuum-valued variables and individuals are located in the continuum space $\mathbb{R}^{n}$.

In opinion formation two major opposing mechanisms are considered: on the one hand, interaction between individuals leading to some type of consensus, and on the other hand, noise that accounts for other factors like self-thinking, media, etc. The balance between these two antagonist effects is key to the long-time evolution of opinions and the formation of large-scale patterns like, for example, emergence of clusters. For instance Ref. 5 investigates the formation of clusters under the rule that interacting individuals adopt the average value of their opinions. Here, we also study the balance between these two opposing effects, particularly, we give conditions for consensus to emerge and we study how given interaction rates affect the speed at which consensus is reached.

We will start first with an extension of the Deffuant-Weisbuch model. ${ }^{15}$ Such models are applied to the study of voters' intentions and their distance correlations. ${ }^{8,9}$ For the asymptotic analysis, we consider the individual-based model presented in Ref. 10 (spatially heterogeneous version of Ref. 15) with an interaction rate inspired from Refs. 29 and 30. In Ref. 10 the authors consider that the interaction rate between individuals is given by a centered Gaussian evaluated at the distance between the opinions of the two interacting individuals. The authors showed that a phase transition emerges between social dissension and a socially cohesive phase with the mean opinion obeying a diffusion equation at the kinetic level. Here we focus our attention on the weak consensus approximation (i.e. the case where little consensus of opinions is reached after interactions). The equilibria and phase transitions that we obtain are consistent with the results in Ref. 10.

There exist related works in the literature that present different settings from ours. In Ref. 21 a similar model, introduced by Ref. 34, is studied with a constant interaction rate and bounded domain. In Ref. 25, the authors investigate a kinetic equation close to ours on a periodic domain. In Ref. 34, the author considers a model where the outcome of a binary interaction depends on each of the individuals' intention but not on their difference of opinions. Finally, in Ref. 14 the authors consider a model for wealth dynamics where binary interactions are possible only if the outcome wealth remains positive.

The paper is structured as follows. In Sec. 2, we discuss the main result, namely Theorem 4.1 where the evolution for the mean opinion dynamics is derived. In Sec. 3 , we present the individual-based model for opinion dynamics in the spatially homogeneous case and consider interactions leading to weak consensus (analogous to the so-called "grazing collision approximation" in gas dynamics). We study the equilibria of these dynamics. Finally, in Sec. 4 we consider the spatially heterogeneous 
case for the previous model and derive the macroscopic equations for the evolution of the mean opinion.

\section{Discussion of the Main Results}

\subsection{Framework}

In this paper, we consider a model where pairs of individuals interact through their opinions or intentions (3.1). A parameter $\gamma$ measures how close both opinions become after an interaction. We assume here that $\gamma$ is small and therefore little consensus is reached during interactions. We will consider that a noise with mean zero and variance $\Sigma^{2}$ is present in the interactions. Finally, these interactions take place at a given rate depending on how close the opinion of a pair of individuals is. Particularly, the rate is parametrized by $\zeta$ which represents the typical scale at which interactions take place. Under the conditions of Proposition 3.2 we prove that the equilibrium for the corresponding mean-field equation is given by a Gaussian with fixed variance $\sigma^{2}$ (given by $\gamma, \zeta, \Sigma^{2}$ ) and undetermined mean $\varphi$. In particular, we give criteria on $\gamma, \Sigma^{2}$ and $\zeta$ for a phase transition to occur between social consensus and social dissent, see Remark 3.3.

We consider two types of rates given by Eqs. (3.2) and (4.2). The first rate corresponds to symmetric interactions, i.e. the rate at which a pair $(i, j)$ interacts is the same as for the pair $(j, i)$. This rate depends only on the spatial distance and opinion distance between the pair of individuals (i.e. the closer individuals are in space and in opinion, the higher the rate at which they interact). In the other case, the interaction is non-symmetric. In this case, individuals forming a cluster in the space-opinion phase space interact very frequently whereas isolated individuals undergo fewer interactions and when they do interact they tend to interact with the close clusters. If $i$ denotes an isolated individual and $j$ an individual belonging to a close cluster then the influence of $j$ on $i$ will be larger than the influence of $i$ on $j$ by a ratio roughly equal to the size of the cluster. This implies that the isolated individual $i$ changes its opinion toward a value closer to the opinion of individual

$j$, while the opinion of $j$ does not change. This non-symmetric relation is key to explain why this rate gives faster consensus than the symmetric case in regions of low density, as we will explain later.

\subsection{Conservative properties and entropy}

The evolution for the mean opinion, or in the language of Ref. 10 of the "intention field", $\varphi=\varphi(\alpha, t)$ (where $\alpha$ is the spatial variable) is given for both rates in Theorem 4.1 in the asymptotic time limit when interactions become localized. The density $\rho$ is constant in time and the density of opinion $\rho \varphi$ evolves according to

$$
\frac{\partial}{\partial t}(\rho \varphi)=C_{s} \nabla_{\alpha} \cdot\left(\rho^{2} \nabla_{\alpha} \varphi\right), \quad C_{s}:=\gamma D \frac{\zeta^{3}}{\left(2 \sigma_{s}^{2}+\zeta^{2}\right)^{3 / 2}}
$$


for the symmetric case and

$$
\frac{\partial}{\partial t}(\rho \varphi)=\frac{C_{a}}{\rho} \nabla_{\alpha} \cdot\left(\rho^{2} \nabla_{\alpha} \varphi\right), \quad C_{a}:=\gamma D \frac{\zeta^{2}}{\sigma_{a}^{2}+\zeta^{2}},
$$

for the non-symmetric case. From these expressions we investigate conservative and homogenization properties for the respective solutions.

To begin with, notice that, for the symmetric case, the density of opinion $\rho \varphi$ is a conserved quantity. This is expected since this is a conserved quantity at the kinetic level. The opposite holds true for the non-symmetric case: the non-conservation of $\rho \varphi$ at the kinetic level is maintained in the macroscopic dynamics. Nevertheless, we notice that in the non-symmetric case the value for $\rho^{2} \varphi$ is conserved (multiplying Eq. (2.2) by $\rho$ as it is independent of time). Notice that if transport of individuals was taken into account on the kinetic equations this conservation property would most likely not hold (in particular, $\rho$ would become time-dependent).

Moreover, from Eqs. (2.1) and (2.2) we can deduce the following entropy dissipation relations:

$$
\frac{\partial}{\partial t} \int_{\mathbb{R}^{n}} \rho \varphi^{2} d \alpha=-C_{s} \int_{\mathbb{R}^{n}} \rho^{2}\left|\nabla_{\alpha} \varphi\right|^{2} d \alpha
$$

for the symmetric case and

$$
\frac{\partial}{\partial t} \int_{\mathbb{R}^{n}} \rho^{2} \varphi^{2} d \alpha=-C_{a} \int_{\mathbb{R}^{n}} \rho^{2}\left|\nabla_{\alpha} \varphi\right|^{2} d \alpha
$$

for the non-symmetric case. This shows that for both cases the $L^{2}\left(\mathbb{R}^{n}\right)$ (weighted) norms on the left-hand side decrease over time. When the time derivative reaches zero, the right-hand side vanishes which implies that $\nabla_{\alpha} \varphi=0$ a.e., and so $\varphi$ is constant a.e. Therefore, the dynamics tend to homogenize the value of the mean opinion, or intention field, $\varphi$. Precisely absolute consensus takes place when $\varphi$ is constant, i.e. there is no difference in the mean opinion between different spatial regions. Observe that, indeed, constant functions are stationary solutions to both equations. In the next section, we investigate for which one of the two rates considered consensus is reached faster asymptotically.

Remark 2.1. (Conserved quantities and analogy with non-equilibrium thermodynamics) Notice that the mean intention $\varphi$ is not a conserved quantity, only the density of intention $\rho \varphi$ is (in the symmetric case). Also, Eq. (2.1) is consistent with Önsager's formalism of non-equilibrium thermodynamics where the time derivatives of the extensive variables (here the mean density of intention) are balanced by the divergence of fluxes that are linear in the gradients of the intensive variables (here the mean intention itself). This is often referred to as the linear flux-force theory. There exists a duality between intensive and extensive variables through the entropy of the system. ${ }^{16}$ This is why, in order to study conservative properties and entropy dissipation relations, we formulate Eqs. (2.1) and (2.2) in Önsager's formalism, ${ }^{16}$ i.e. by considering the time derivative corresponding to the extensive variable $\rho \varphi$ instead of that of the intensive variable $\varphi$. However, in the non-symmetric case, the 
formalism of non-equilibrium thermodynamics does not strictly apply since the time derivative of the density of intention $\rho \varphi$ is not balanced by a divergence. Instead, there is the pre-factor $1 / \rho$ in front of the divergence which makes the equation non-conservative. Thus, in this case, the standard entropy $\int \rho \varphi^{2} d x$ as put forward by Önsager need not be dissipated. But another entropy $\int \rho^{2} \varphi^{2} d x$ is.

To conclude this section, we remark that by Proposition 3.2 the diffusion constants can be rewritten as $C_{s}=\gamma D\left(\sqrt{\zeta^{2}-\kappa} / \zeta\right)^{3}$ and $C_{a}=\gamma D\left(2 \zeta^{2}-\kappa\right) /\left(2 \zeta^{2}\right)$ (with $\kappa=\Sigma^{2} / \gamma$ ) and so they stay positive for the same range values of $\kappa$ as that for which the Gaussian equilibria given in Proposition 3.2 is defined. This shows that the final model is well-posed.

\subsection{Comparison in the speed of consensus}

To compare the speed of consensus given by the two different rates, we recast again Eqs. (4.12)-(4.13) from Theorem 4.1 into, assuming that $\rho>0$,

$$
\frac{\partial \varphi}{\partial t}-C_{s}\left(\rho \Delta_{\alpha} \varphi+2 \nabla_{\alpha} \rho \cdot \nabla_{\alpha} \varphi\right)=0, \quad C_{s}:=\gamma D \frac{\zeta^{3}}{\left(2 \sigma_{s}^{2}+\zeta^{2}\right)^{3 / 2}},
$$

for the symmetric case and

$$
\frac{\partial \varphi}{\partial t}-C_{a}\left(\Delta_{\alpha} \varphi+\frac{2}{\rho} \nabla_{\alpha} \rho \cdot \nabla_{\alpha} \varphi\right)=0, \quad C_{a}:=\gamma D \frac{\zeta^{2}}{\sigma_{a}^{2}+\zeta^{2}},
$$

for the non-symmetric case $\left(\sigma_{s}\right.$ and $\sigma_{a}$ are given in Proposition 3.2). One can check that $C_{a}>C_{s}$. In both cases we have that the mean opinion, or intention field, diffuses and is transported in the direction $-\nabla_{\alpha} \rho$, i.e. from places of high concentration of individuals to places of low concentration. Since absolute consensus takes place when $\varphi$ is constant, the faster the mean opinion $\varphi$ is diffused and transported through space, the faster consensus is reached.

We start by examining regions with low population, say $\rho \leq 1$. We observe that the diffusive coefficient for the symmetric case, given by $C_{s} \rho$, is always smaller than that of the non-symmetric case, corresponding to $C_{a}$ (since $C_{a}>C_{s}$ ). In particular, in this symmetric case, diffusion slows down in regions with lower density of individuals. Also the transport of the mean opinion toward areas of lower density is slower in the symmetric case since this speed corresponds to $C_{s}$ whereas in the non-symmetric case it corresponds to $C_{a} / \rho$. Notice then that, in the non-symmetric case, in low density regions this transport takes place very fast. However in the nonsymmetric case, interaction rates are scale-invariant with the local density while in the symmetric case they are homogeneous of degree one with the density. So, in the non-symmetric case, agents in low density regions interact at the same rate as in the high density regions while in the symmetric case, they interact at a much lower rate. The effect is opposite in the large density regions.

In conclusion, from Theorem 4.1 we deduce the following.

Corollary 2.1. Low density regions reach consensus faster in the non-symmetric rate case. By contrast, the large density regions reach consensus faster in the 
symmetric case. The crossover density between these two behaviors is given by $C_{s} \rho=C_{a}$, i.e.

$$
\rho=\frac{C_{a}}{C_{s}}
$$

This crossover in the speed to consensus can be understood heuristically as follows. In the symmetric case any pair of individuals has the same influence on each other. Contrary to this, in the non-symmetric case, individuals belonging to a cluster (high density region) influence the opinion of isolated individuals (low density regions) but not the other way around, this is precisely due to the non-symmetric nature of the rate. As a consequence, in the non-symmetric case, because clusters have more influence on isolated individuals, isolated individuals adopt quickly the opinion of the cluster. This can be seen by examining the role of the convection term (the third term on the left-hand side of (2.5) and (2.6)). Indeed this convection term shows a transport of the mean opinion in the direction of the gradient for $\rho$, i.e. from places of high concentration to places of low concentration. Actually this convection takes place much faster in low density regions in the non-symmetric case than in the symmetric case. Now, in high density regions, in the non-symmetric case, individuals of a cluster tend to interact very frequently among themselves but they interact less frequently with members of other clusters than in the symmetric case. This is why for higher density regions the symmetric rate reaches consensus faster: there are more interactions (exchange of opinions) between clusters.

\section{Rate Model and Weak Consensus Approximation}

\subsection{Kinetic model: Symmetric and non-symmetric cases}

We first consider a spatially homogeneous model for opinion formation. Denote by $\phi_{i}(t) \in \mathbb{R}$ the opinion of agent $i$ at time $t$; for example, it could represent the intention of agent $i$ to vote for a particular party as in Ref. 10. When the pair of agents $(i, j)$ interact, they exchange opinions according to the following rule $\left(\phi_{i}, \phi_{j}\right) \rightarrow\left(\phi_{i}^{\prime}, \phi_{j}\right)$ with

$$
\phi_{i}^{\prime}=\phi_{i}+\gamma\left(\phi_{j}-\phi_{i}\right)+\eta_{i}
$$

where $0 \leq \gamma \leq \frac{1}{2}$ is a constant which measures how opinions get closer after an interaction takes place. For each $i=1, \ldots, N$, where $N$ is the number of agents, $\left(\eta_{i}\right)_{i=1, \ldots, N}$ are independent, identically distributed random variables distributed according to a probability density $q=q(\eta)$ with zero mean, variance $\Sigma^{2}$ and decaying moments. This noise source accounts for other influences. The interaction rate between a pair of individuals, given by $G_{\zeta}(\phi)=G\left(\left|\phi_{i}-\phi_{j}\right| / \zeta\right)$, is dependent on the distance between their opinions and is a decreasing function of its argument. In other words, individuals with far-away opinions interact at a lower rate (as in Ref. 15). The parameter $\zeta$ is a typical scale in the sense that individuals with opinions distant by a quantity greater than $\zeta$ have a probability close to zero to interact. 
Here, we will consider two types of interaction rates which we write compactly as

$$
\frac{G_{\zeta}\left(\left|\phi_{i}-\phi_{j}\right|\right)}{\mathrm{H}\left(\frac{1}{N} \sum_{k=1}^{N} G_{\zeta}\left(\left|\phi_{i}-\phi_{k}\right|\right)\right)},
$$

where $\mathrm{H}$ is a function that can correspond to either the constant 1 or the identity operator depending on the case we are interested in:

$$
\begin{aligned}
& \mathrm{H}: g \in[0, \infty) \rightarrow \mathrm{H}(g) \in[0, \infty),
\end{aligned}
$$

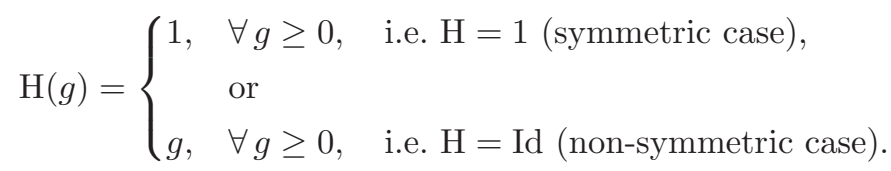

If $\mathrm{H} \equiv 1$, then the rate of interactions between two agents is symmetric. On the contrary, if $\mathrm{H}=\mathrm{Id}$, the rate of interactions for pair $(i, j)$ is not the same as for pair $(j, i)$. In particular, the non-symmetric rate case is inspired by the models in Refs. 29 and 30.

We denote by $f=f(\phi, t)$ the probability distribution of agent's intentions $\phi$ at time $t$. According to the previous rule, and assuming propagation of chaos (see Remark 3.2 and Ref. 13), the evolution of $f$ is given for any test function $g$, by

$$
\begin{aligned}
\frac{d}{d t} \int_{\mathbb{R}} f g d \phi= & \int_{\mathbb{R}^{3}}[g(\phi+\gamma(\psi-\phi)+\eta)-g(\phi)] \\
& \times f(\phi, t) f(\psi, t) \frac{G_{\zeta}(|\phi-\psi|)}{\mathrm{H}\left(G_{\zeta} * f\right)} q(\eta) d \eta d \phi d \psi
\end{aligned}
$$

Remark 3.1. The original interaction model presented in Ref. 10 considers the symmetric interaction rate with interaction rules $\left(\phi_{i}, \phi_{j}\right) \rightarrow\left(\phi_{i}^{\prime}, \phi_{j}^{\prime}\right)$ given by:

$$
\begin{aligned}
\phi_{i}^{\prime} & =\phi_{i}+\gamma\left(\phi_{j}-\phi_{i}\right)+\eta_{i}, \\
\phi_{j}^{\prime} & =\phi_{j}+\gamma\left(\phi_{i}-\phi_{j}\right)+\eta_{j},
\end{aligned}
$$

$0 \leq \gamma \leq \frac{1}{2}$. However, the kinetic equation for this system corresponds precisely to (3.3) (up to a factor 2 due to the rate being counted twice). This is precisely a consequence of the symmetry of the interactions. Therefore, for the following analysis one can consider either of the two interacting processes.

\subsection{Approximation under weak consensus assumption}

Next we assume that the consensus parameter $\gamma$ is small, meaning that there is weak consensus during interactions. This is analogous to the so-called "grazing collisions" in gas dynamics. ${ }^{18}$ To prevent the noise term to dominate the dynamics, we assume too that $\Sigma^{2}$ is also small and of the same order of magnitude. In particular, we define 
$\kappa$ as

$$
\Sigma^{2}=\kappa \gamma, \quad \kappa=\text { Constant. }
$$

Under these assumptions, we Taylor expand the bracket in (4.2) to obtain

$$
\begin{aligned}
\frac{d}{d t} \int_{\mathbb{R}} f g d \phi= & \gamma \int_{\mathbb{R}^{2}}\left[g^{\prime}(\phi)(\psi-\phi)+\frac{\kappa}{2} g^{\prime \prime}(\phi)\right] \\
& \times f(\phi, t) f(\psi, t) \frac{G_{\zeta}(|\phi-\psi|)}{\mathrm{H}\left(G_{\zeta} * f\right)} d \phi d \psi,
\end{aligned}
$$

where we have dropped the terms $\mathcal{O}\left(\gamma^{2}\right), \mathcal{O}\left(\Sigma^{2}\right)$ and the ones with higher moments of $q$. The equation for $f$ obtained after dropping the low-order terms approximates the evolution of the opinion dynamics under the weak consensus assumption. This is in line with the grazing collision approximation in gas dynamics.

Using integration by parts, we get the strong form of the Fokker-Planck equation for $f$ :

$$
\begin{aligned}
\frac{\partial f}{\partial t} & =Q(f) \\
Q(f) & =\gamma \partial_{\phi}\left\{\frac{\left(\phi G_{\zeta}\right) * f}{\mathrm{H}\left(G_{\zeta} * f\right)} f+\frac{\kappa}{2} \partial_{\phi}\left(\frac{\left(G_{\zeta} * f\right)}{\mathrm{H}\left(G_{\zeta} * f\right)} f\right)\right\} .
\end{aligned}
$$

Remark 3.2. We can directly derive this equation from a stochastic interacting particle system. We introduce the following system for the intention $\phi_{i}(t)$ of agent $i$ :

$$
\begin{aligned}
d \phi_{i}= & \gamma\left[\frac{\frac{1}{N} \sum_{j=1}^{N}\left(\phi_{j}-\phi_{i}\right) G_{\zeta}\left(\phi_{j}-\phi_{i}\right)}{\mathrm{H}\left(\frac{1}{N} \sum_{k=1}^{N} G_{\zeta}\left(\left|\phi_{i}-\phi_{j}\right|\right)\right)}\right] d t \\
& +\sqrt{\gamma \kappa\left(\frac{\frac{1}{N} \sum_{j=1}^{N} G_{\zeta}\left(\phi_{j}-\phi_{i}\right)}{\mathrm{H}\left(\frac{1}{N} \sum_{k=1}^{N} G_{\zeta}\left(\left|\phi_{i}-\phi_{j}\right|\right)\right)}\right)} d B_{t}^{i},
\end{aligned}
$$

where $B_{t}^{i}$ are independent standard Brownian motions. We assume that the initial data $\phi_{i}(0)$ are drawn independently out of identical probability densities $f_{0}(\phi)$. We notice that the system is invariant under permutations of indices $i$. Hence the $\phi_{i}(t)$ are indistinguishable. Introducing the one- and two-particle marginal distributions $f_{N}^{(1)}(\phi)$ and $f_{N}^{(2)}(\phi, \psi)$, and denoting by $g(\phi)$ any test function, we have

$$
\begin{aligned}
\frac{d}{d t} \int_{\mathbb{R}} g(\phi) f_{N}^{(1)}(\phi, t) d \phi= & \gamma \frac{N-1}{N} \int_{\mathbb{R}^{2}}\left[g^{\prime}(\phi)(\psi-\phi)+\frac{\kappa}{2} g^{\prime \prime}(\phi)\right] \\
& \times \frac{G_{\zeta}(\psi-\phi)}{\mathrm{H}\left(G_{\zeta} * f\right)} f_{N}^{(2)}(\phi, \psi, t) d \phi d \psi
\end{aligned}
$$

Assuming propagation of chaos in the limit $N \rightarrow \infty$, namely there exists a one-particle distribution $f(\phi, t)$ such that $f_{N}^{(1)}(\phi, t) \rightarrow f(\phi, t)$ and $f_{N}^{(2)}(\phi, \psi, t) \rightarrow$ 
$f(\phi, t) f(\psi, t)$, we get

$$
\begin{aligned}
\frac{d}{d t} \int_{\mathbb{R}} g(\phi) f(\phi, t) d \phi= & \gamma \int_{\mathbb{R}^{2}}\left[g^{\prime}(\phi)(\psi-\phi)+\frac{\kappa}{2} g^{\prime \prime}(\phi)\right] \\
& \times \frac{G_{\zeta}(\psi-\phi)}{\mathrm{H}\left(G_{\zeta} * f\right)} f(\phi, t) f(\psi, t) d \phi d \psi,
\end{aligned}
$$

which is the same as (3.5) without the low-order terms.

We can write (3.7) in a more convenient way by introducing a potential $V_{f}(\phi)$ such that

$$
\partial_{\phi} V_{f}=\frac{\left(\phi G_{\zeta}\right) * f}{G_{\zeta} * f}
$$

and a Gibbs distribution

$$
M_{f}(\phi)=\frac{1}{Z_{f}} \exp \left(-\frac{2 V_{f}(\phi)}{\kappa}\right), \quad Z_{f}=\int_{\mathbb{R}} \exp \left(-\frac{2 V_{f}(\phi)}{\kappa}\right) d \phi .
$$

We recast (3.7) into

$$
Q(f)=\gamma \frac{\kappa}{2} \partial_{\phi}\left\{M_{f} \partial_{\phi}\left(\frac{\left(G_{\zeta} * f\right)}{\mathrm{H}\left(G_{\zeta} * f\right)} \frac{f}{M_{f}}\right)\right\},
$$

which in the symmetric case $(\mathrm{H} \equiv 1)$ corresponds to

$$
Q(f)=\gamma \frac{\kappa}{2} \partial_{\phi}\left\{M_{f} \partial_{\phi}\left(\frac{\left(G_{\zeta} * f\right) f}{M_{f}}\right)\right\}
$$

and for the non-symmetric case $(\mathrm{H}=\mathrm{Id})$,

$$
Q(f)=\gamma \frac{\kappa}{2} \partial_{\phi}\left\{M_{f} \partial_{\phi}\left(\frac{f}{M_{f}}\right)\right\} .
$$

Remember that $\kappa \gamma=\Sigma^{2}$ which is assumed to be small.

\subsection{Equilibria and phase transition}

In this section, we investigate the equilibria for the operator $Q$ in Eq. (3.10). In the general case, we have the following.

Proposition 3.1. $f_{\mathrm{eq}}$ is an equilibrium, i.e. a solution of $Q\left(f_{\mathrm{eq}}\right)=0$, if and only if $f$ satisfies:

(i) in the symmetric case,

$$
\left(G_{\zeta} * f\right) f=B_{f} M_{f}, \quad B_{f}=\int_{\mathbb{R}}\left(G_{\zeta} * f\right) f d \phi ;
$$

(ii) in the non-symmetric case,

$$
f=M_{f}
$$


Proof. Integration by parts leads to

$$
\int_{\mathbb{R}} Q(f) \frac{\left(G_{\zeta} * f\right)}{\mathrm{H}\left(G_{\zeta} * f\right)} \frac{f}{M_{f}} d \phi=-\gamma \frac{\kappa}{2} \int_{\mathbb{R}} M_{f}\left|\partial_{\phi}\left(\frac{\left(G_{\zeta} * f\right)}{\mathrm{H}\left(G_{\zeta} * f\right)} \frac{f}{M_{f}}\right)\right|^{2} d \phi .
$$

Then $Q(f)=0$ if and only if $\frac{\left(G_{\zeta} * f\right)}{\mathrm{H}\left(G_{\zeta} * f\right)} \frac{f}{M_{f}}$ is a constant. This constant is given by the constraint $\int M_{f}=1$.

Proving the existence of a fixed point for Eqs. (3.12) and (3.13) is in general challenging. We will focus our attention on the Gaussian case where

$$
G(u)=e^{-u^{2} / 2} .
$$

In this case, we have the following.

Proposition 3.2. Assume that the interaction rate $G$ is given by (3.14). Then Gaussian functions $f_{\mathrm{eq}}=F_{\sigma, \varphi}$, with

$$
F_{\sigma, \varphi}(\phi)=\frac{1}{\sqrt{2 \pi} \sigma} \exp \left(-\frac{|\phi-\varphi|^{2}}{2 \sigma^{2}}\right)
$$

are equilibria, provided that:

(i) in the symmetric case, $\sigma=\sigma_{s}$ with

$$
\sigma_{s}^{2}=\frac{\zeta^{2}}{2\left(\frac{\zeta^{2}}{\kappa}-1\right)}
$$

(ii) in the non-symmetric case, $\sigma=\sigma_{a}$ with

$$
\sigma_{a}^{2}=\frac{\zeta^{2}}{\frac{2 \zeta^{2}}{\kappa}-1} .
$$

Therefore, Gaussian equilibria exist only for $\kappa$ such that:

(i) in the symmetric case,

$$
\kappa<\kappa_{c, s}(\zeta)=\zeta^{2}
$$

(ii) in the non-symmetric case,

$$
\kappa<\kappa_{c, a}(\zeta)=2 \zeta^{2}
$$

Remark 3.3. (Constraints and phase transition)

- The constraints in Eqs. (3.18) and (3.19) give a critical value of $\kappa$ for which a phase transition takes place between existence and non-existence of Gaussian equilibria. Notice that when $\kappa$ approaches its critical value the variance of the equilibria diverges. Therefore, opinions spread toward dissension. Otherwise, a consensus arises. On the contrary, if the noise strength $\Sigma^{2} \rightarrow 0$ then $\sigma \rightarrow 0$ and we expect to converge to a Dirac-delta distribution. The phase transition that we observe here corresponds to the one proven in Ref. 10 when considering the grazing collision case. 
- It is easy to check that $\sigma_{s}>\sigma_{a}$, therefore, opinions in the symmetric case are spread more around their mean than in the non-symmetric one.

- Replacing the value of $\kappa$ in Eq. (3.4) we can rewrite Eqs. (3.18) and (3.19) as

$$
\Sigma^{2}<c_{0} \zeta^{2} \gamma
$$

for $c_{0}=1$ in the symmetric case and $c_{0}=2$ in the non-symmetric case. Notice that for higher values of $\gamma$ and $\zeta$, we expect more consensus and, therefore, condensation of the opinions; the larger $\gamma$ is, the closer the agents get in their opinions and the larger the typical interaction range $\zeta$ is, the more interactions take place and the faster a consensus can be reached. The product of these two quantities must bound the strength of the noise $\Sigma^{2}$.

Notice that in Ref. 10 the constraint for the symmetric case takes the form

$$
\Sigma^{2}<\zeta^{2} \gamma(1-\gamma)
$$

This is consistent with our result as in Sec. 3.2 we assumed weak consensus interactions and drop terms of order $\gamma^{2}$.

In the sequel we will use repeatedly the following identities (expressed in the notation of Eq. (3.15)):

$$
\begin{aligned}
F_{\sigma, 0} F_{\eta, 0} & =\frac{1}{\sqrt{2 \pi\left(\sigma^{2}+\eta^{2}\right)}} F \sqrt{\frac{\sigma^{2} \eta^{2}}{\sigma^{2}+\eta^{2}}}, 0 \\
F_{\sigma, \varphi} * F_{\eta, \mu} & =F \sqrt{\sigma^{2}+\eta^{2}}, \varphi+\mu \\
\partial_{\varphi}(f * g) & =\left(\partial_{\varphi} f * g\right)=\left(f * \partial_{\varphi} g\right) .
\end{aligned}
$$

Notice that in the Gaussian case (3.14) we can express $G_{\zeta}$ as

$$
G_{\zeta}=\sqrt{2 \pi} \zeta F_{\zeta, 0}
$$

Proof of Proposition 3.2. In the case (3.14) we have $\phi G_{\zeta}=-\zeta^{2} \partial_{\phi} G_{\zeta}$ and so

$$
\frac{\left(\phi G_{\zeta}\right) * f}{G_{\zeta} * f}=-\zeta^{2} \partial_{\phi} \log \left(G_{\zeta} * f\right)
$$

which implies that

$$
V_{f}=-\zeta^{2} \log \left(G_{\zeta} * f\right)+\text { Constant }
$$

This implies that

$$
M_{f}=\frac{1}{C_{f}}\left(G_{\zeta} * f\right)^{\frac{2 \zeta^{2}}{\kappa}}, \quad C_{f}=\int_{\mathbb{R}}\left(G_{\zeta} * f\right)^{\frac{2 \zeta^{2}}{\kappa}} d \phi .
$$

For the symmetric case, inserting this into (3.12), we get

$$
\left(G_{\zeta} * f\right) f=\frac{B_{f}}{C_{f}}\left(G_{\zeta} * f\right)^{\frac{2 \zeta^{2}}{\kappa}},
$$


or

$$
f=\frac{B_{f}}{C_{f}}\left(G_{\zeta} * f\right)^{\frac{2 \zeta^{2}}{\kappa}-1} .
$$

Now, one can check using (3.21) and (3.23) that $f=F_{\sigma, \varphi}$ is a solution provided that

$$
\sigma^{2}=\frac{\zeta^{2}+\sigma^{2}}{\frac{2 \zeta^{2}}{\kappa}-1}
$$

which leads to (3.16). The condition for $\sigma^{2}>0$ is that $\frac{\zeta^{2}}{\kappa}-1>0$ which leads to the constraint $\kappa<\zeta^{2}$.

The non-symmetric case is dealt with analogously by inserting expression (3.24) into (3.13).

\section{Space Inhomogeneous Model and Continuum Limit}

In this section, we consider the space inhomogeneous version of the opinion formation model and investigate the evolution of the mean opinion dynamic when interactions become localized in space. This is done in the spirit of hydrodynamic limits for kinetic equations.

\subsection{Derivation of the kinetic model}

In this section, we assume that the agents are endowed with a spatial variable $\alpha \in \mathbb{R}^{n}$ (with $n \in\{1,2,3\}$ ) which can be the geographical distance or any other social metric. Then, each agent labeled $i(i \in\{1, \ldots, N\})$ is described by its location variable $\alpha_{i}(t)$ and its opinion $\phi_{i}(t)$ at time $t$. Inspired by Ref. 10, we can interpret $\alpha_{i}$ as the city of voter $i$. It interacts with voter $j$ with opinion $\phi_{j}$ and resident in city $\alpha_{j}$ according to rule (3.1) with rate

$$
\frac{G_{\zeta}\left(\phi_{i}-\phi_{j}\right) F_{\varepsilon}\left(\alpha_{i}-\alpha_{j}\right)}{\mathrm{H}\left(\frac{1}{N} \sum_{k=1}^{N} G_{\zeta}\left(\phi_{i}-\phi_{k}\right) F_{\varepsilon}\left(\alpha_{i}-\alpha_{k}\right)\right)},
$$

with

$$
G_{\zeta}(\phi)=G\left(\frac{|\phi|}{\zeta}\right), \quad F_{\varepsilon}(\alpha)=\frac{1}{\varepsilon^{n}} F\left(\frac{|\alpha|}{\varepsilon}\right),
$$

and $H$ given in Eq. (3.2). The parameter $\varepsilon$ is supposed to be a measure of the interaction range in the position variable $\alpha$. We subsequently normalize $F$ such that

$$
\int_{\mathbb{R}^{n}} F(|\alpha|) d \alpha=1
$$

and denote by $D$ the constant

$$
D=\frac{1}{2 n} \int_{\mathbb{R}^{n}} F(|\alpha|)|\alpha|^{2} d \alpha
$$

Let $f=f(\alpha, \phi, t)$ be the probability density of the agents in the $(\alpha, \phi)$ space at time $t$. Following the same reasoning as previously, we can write for any test 
function $g=g(\alpha, \phi)$ :

$$
\begin{aligned}
\frac{d}{d t} \int_{\mathbb{R}^{n+1}} f g d \alpha d \phi= & \int_{\mathbb{R}^{2 n+3}}[g(\alpha, \phi+\gamma(\psi-\phi)+\eta)-g(\alpha, \phi)] f(\alpha, \phi, t) f(\beta, \psi, t) \\
& \times \frac{G_{\zeta}(|\phi-\psi|)}{\mathrm{H}\left[F_{\varepsilon} G_{\zeta} *_{\alpha, \phi} f\right](\phi)} F_{\varepsilon}(|\alpha-\beta|) q(\eta) d \eta d \phi d \psi d \alpha d \beta
\end{aligned}
$$

As done previously in Sec. 3.2 for the spatially homogeneous case, we consider the case of weak consensus interactions where $\gamma$ and $\Sigma^{2}$ are small and of the same order of magnitude. Taylor expanding the bracket in the previous expression and dropping the low-order terms we get

$$
\begin{aligned}
\frac{d}{d t} \int_{\mathbb{R}} f g d \alpha d \phi= & \gamma \int_{\mathbb{R}^{2 n+2}}\left[\partial_{\phi} g(\alpha, \phi)(\psi-\phi)+\frac{\kappa}{2} \partial_{\phi}^{2} g(\alpha, \phi)\right] f(\alpha, \phi, t) f(\beta, \psi, t) \\
& \times \frac{G_{\zeta}(|\phi-\psi|)}{\mathrm{H}\left[F_{\varepsilon} G_{\zeta} *_{\alpha, \phi} f\right](\phi)} F_{\varepsilon}(|\alpha-\beta|) d \phi d \psi d \alpha d \beta,
\end{aligned}
$$

where $\kappa$ is given by Eq. (3.4).

Using integration by parts, we get the strong form of the Fokker-Planck equation for $f$ in the inhomogeneous case:

$$
\begin{aligned}
\frac{\partial f}{\partial t} & =Q(f), \\
Q(f) & =\gamma \partial_{\phi}\left\{\frac{\left(F_{\varepsilon} \phi G_{\zeta}\right) *_{\alpha, \phi} f}{\mathrm{H}\left[F_{\varepsilon} G_{\zeta} *_{\alpha, \phi} f\right]} f+\frac{\kappa}{2} \partial_{\phi}\left(\frac{\left(F_{\varepsilon} G_{\zeta}\right) *_{\alpha, \phi} f}{\mathrm{H}\left[F_{\varepsilon} G_{\zeta} *_{\alpha, \phi} f\right]} f\right)\right\},
\end{aligned}
$$

where $*_{\alpha, \phi}$ denotes a convolution in both the $\alpha$ and $\phi$ variables. This system can also be directly derived from a stochastic differential system in exactly the same manner as in the homogeneous case, Remark 3.2. Details are omitted.

Now, we plan to investigate the $\varepsilon \rightarrow 0$ limit, i.e. we assume that the exchange of intentions is at leading order a local phenomenon in space. We note that for any arbitrary function $\varphi$, we have

$$
F_{\varepsilon} * \varphi=\varphi+\varepsilon^{2} D \Delta_{\alpha} \varphi+\mathcal{O}\left(\varepsilon^{4}\right),
$$

where $\Delta_{\alpha}$ denotes the Laplacian operator in the $\alpha$ variable. Using this expansion and introducing a diffusive time variable $t^{\prime}=\varepsilon^{2} t$, we get, dropping the primes,

$$
\frac{\partial f^{\varepsilon}}{\partial t}+\mathrm{R}^{\varepsilon}=\frac{1}{\varepsilon^{2}} Q\left(f^{\varepsilon}\right)
$$

where the operator $Q$ is given in (3.7) and:

(i) for the symmetric case,

$$
\mathrm{R}^{\varepsilon}=\mathrm{R}_{s}^{\varepsilon}=-\gamma D \partial_{\phi}\left\{\left(\phi G_{\zeta} * \Delta_{\alpha} f^{\varepsilon}\right) f^{\varepsilon}+\frac{\kappa}{2} \partial_{\phi}\left(\left(G_{\zeta} * \Delta_{\alpha} f^{\varepsilon}\right) f^{\varepsilon}\right)\right\} ;
$$

(ii) for the non-symmetric case,

$$
\mathrm{R}^{\varepsilon}=\mathrm{R}_{a}^{\varepsilon}=-\gamma D \partial_{\phi}\left\{\frac{\left(\phi G_{\zeta}\right) * f}{\left(G_{\zeta} * f\right)^{2}} f\left(G_{\zeta} * \Delta_{\alpha} f\right)-\frac{\left(\phi G_{\zeta}\right) * \Delta_{\alpha} f}{G_{\zeta} * f} f\right\} .
$$


In both cases $*$ denotes a convolution with respect to the $\phi$ variable only. In the following section, we study the formal limit as $\varepsilon \rightarrow 0$ for the Gaussian case (3.14).

\subsection{Derivation of the hydrodynamic model (Gaussian case)}

In this section, we restrict ourselves to the Gaussian case (3.14) and study the evolution of the mean opinion for Eq. (4.6) in the limit $\varepsilon \rightarrow 0$. To compute this limit, the concept of collision-invariant is key as it will be explained next. However, in the non-symmetric case the mean opinion is not a conserved quantity. This difficulty will be overcome by using a technique reminiscent of the classical Hilbert expansion. ${ }^{13}$ Notice that the lack of conserved quantities is not new in the literature, see Ref. 17 where the authors use a novel technique to compute the hydrodynamic limit, different from what we do here.

First, we deduce the following.

Lemma 4.1. Assume that $G_{\zeta}$ is given by (3.23) with $\kappa$ satisfying (3.18) in the symmetric case and (3.19) in the non-symmetric case. Assume, further, that the Gaussian equilibria given by Proposition 3.2 are the only equilibria of $Q$. Let $f^{\varepsilon}$ be a solution of (4.6) and suppose that $f^{\varepsilon} \rightarrow f$ as nicely as needed. Then, there exist two functions $\rho=\rho(\alpha, t)$ and $\varphi=\varphi(\alpha, t)$ such that

$$
f(\alpha, \phi, t)=\rho(\alpha, t) F_{\sigma, \varphi(\alpha, t)}(\phi) .
$$

The functions $\rho=\rho(\alpha, t)$ and $\varphi=\varphi(\alpha, t)$ are respectively the agents' density and intention field at location $\alpha$ and time $t$, i.e. we have

$$
\int_{\mathbb{R}} f(\alpha, \phi, t) d \phi=\rho(\alpha, t), \quad \int_{\mathbb{R}} f(\alpha, \phi, t) \phi d \phi=\rho(\alpha, t) \varphi(\alpha, t) .
$$

Proof. Letting $\varepsilon \rightarrow 0$ in (4.6), we get that $f$ formally satisfies $Q(f)=0$. Since $Q$ only operates with respect to the $\phi$ variable we deduce that $f$ is proportional to $F_{\sigma, \varphi}$ by Lemma 3.2 with $\varphi$ possibly depending on $(\alpha, t)$. Furthermore, since $F_{\sigma, \varphi}$ is a probability distribution with respect to $\phi$, the proportionality coefficient is the density $\rho(\alpha, t)$.

Remark 4.1. (The general case) Notice that the equilibria defined via Eqs. (3.12) and (3.13) (if they exist) are not necessarily defined by some moments of $f$ like the total mass, mean value or variance. This is the case in classical kinetic theory where the goal in the hydrodynamic limit is to find the evolution of these moments, which correspond also to conserved quantities of the system. However, here, in the general case, it is not clear that this is the case. A partial attempt to solve this problem can be found in Ref. 22 . 
The function (4.9) is a local thermodynamical equilibrium of the system. Now, we need to find the collision invariants of $Q$, namely the functions $\chi(\phi)$ such that

$$
\int_{\mathbb{R}} Q(f)(\phi) \chi(\phi) d \phi=0, \quad \forall f .
$$

We have the following.

Lemma 4.2. (Collision invariants) The function $\chi(\phi)=1$ is a collision-invariant of the symmetric and non-symmetric cases. The function $\chi(\phi)=\phi$ is a collisioninvariant of the symmetric case.

Proof. Since $Q$ is a derivative with respect to $\phi$, by integration by parts, it is straightforward that $\chi(\phi)=1$ is a collision-invariant for both cases. Now, for the symmetric case, considering $\chi(\phi)=\phi$, we have, using integration by parts,

$$
\int_{\mathbb{R}} Q(f)(\phi) \phi d \phi=-\gamma \int_{\mathbb{R}^{2}}(\phi-\psi) G_{\zeta}(\phi-\psi) f(\phi) f(\psi) d \phi d \psi=0,
$$

because the function $(\phi, \psi) \rightarrow G_{\zeta}(\psi-\phi) f(\phi) f(\psi)$ is invariant by exchange of $\phi$ and $\psi$ while the function $(\phi, \psi) \rightarrow(\psi-\phi)$ is changed in its opposite.

That $\chi(\phi)=1$ and $\chi(\phi)=\phi$ are collision invariants corresponds to the conservation of the number of agents and that of the mean intention during an encounter, respectively.

Lemma 4.3. (Collision-invariant for the linearized operator) Assume that the solution to (4.4) decays sufficiently fast as $|\phi| \rightarrow \infty$. Then, under the assumptions of Lemma 4.1, in the non-symmetric case, the primitive $\chi(\phi)=\int^{\phi}\left(G_{\zeta} *\right.$ $\left.F_{\sigma, \varphi(\alpha, t)}\right)\left(\phi^{\prime}\right) d \phi^{\prime}$ is a collision-invariant of the linearized collision operator, denoted $\operatorname{Lin}_{Q}$, around $F_{\sigma, \varphi(\alpha, t)}$.

Proof. The linearized form of $Q$ in Eq. (3.11) around $F_{\sigma, \varphi(\alpha, t)}$ is given by:

$$
\begin{aligned}
\operatorname{Lin}_{Q}(f)= & \gamma \partial_{\phi}\left(\frac{\left(\phi G_{\zeta} * f\right) F_{\sigma, \varphi(\alpha, t)}}{G_{\zeta} * F_{\sigma, \varphi(\alpha, t)}}+\frac{\left(\phi G_{\zeta} * F_{\sigma, \varphi(\alpha, t)}\right) f}{G_{\zeta} * F_{\sigma, \varphi(\alpha, t)}}\right. \\
& \left.-\frac{\left(\phi G_{\zeta} * F_{\sigma, \varphi(\alpha, t)}\right)\left(G_{\zeta} * f\right) F_{\sigma, \varphi(\alpha, t)}}{\left(G_{\zeta} * F_{\sigma, \varphi(\alpha, t)}\right)^{2}}+\frac{\kappa}{2} \partial_{\phi} f\right) .
\end{aligned}
$$

By assumption, the boundary terms vanish. Integrating against $\chi$ and using that $\chi$ is the primitive of $G_{\zeta} * F_{\sigma, \varphi(\alpha, t)}$, we obtain

$$
\begin{aligned}
\int_{\mathbb{R}} \operatorname{Lin}_{Q}(f)(\phi)\left[\int^{\phi}\left(G_{\zeta} * F_{\sigma, \varphi(\alpha, t)}\right)\left(\phi^{\prime}\right) d \phi^{\prime}\right] d \phi \\
=-\gamma \int_{\mathbb{R}}\left(\frac{\left(\phi G_{\zeta} * f\right) F_{\sigma, \varphi(\alpha, t)}}{G_{\zeta} * F_{\sigma, \varphi(\alpha, t)}}+\frac{\left(\phi G_{\zeta} * F_{\sigma, \varphi(\alpha, t)}\right) f}{G_{\zeta} * F_{\sigma, \varphi(\alpha, t)}}\right.
\end{aligned}
$$




$$
\left.-\frac{\left(\phi G_{\zeta} * F_{\sigma, \varphi(\alpha, t)}\right)\left(G_{\zeta} * f\right) F_{\sigma, \varphi(\alpha, t)}}{\left(G_{\zeta} * F_{\sigma, \varphi(\alpha, t)}\right)^{2}}+\frac{\kappa}{2} \partial_{\phi} f\right)(\phi)\left(G_{\zeta} * F_{\sigma, \varphi(\alpha, t)}\right)(\phi) d \phi
$$

(Here we use Fubini's theorem to change the order of integration...)

$$
\begin{aligned}
= & -\gamma \int_{\mathbb{R}} f\left[-\left(\phi G_{\zeta} * F_{\sigma, \varphi(\alpha, t)}\right)(\phi)+\left(\phi G_{\zeta} * F_{\sigma, \varphi(\alpha, t)}\right)(\phi)\right. \\
& \left.-G_{\zeta} *\left(\frac{\left(\phi G_{\zeta} * F_{\sigma, \varphi(\alpha, t)}\right) F_{\sigma, \varphi(\alpha, t)}}{\left(G_{\zeta} * F_{\sigma, \varphi(\alpha, t)}\right)}\right)-\frac{\kappa}{2} \partial_{\phi}\left(G_{\zeta} * F_{\sigma, \varphi(\alpha, t)}\right)(\phi)\right] d \phi \\
= & -\gamma \int_{\mathbb{R}} f\left[-G_{\zeta} *\left(\frac{\left(\phi G_{\zeta} * F_{\sigma, \varphi(\alpha, t)}\right) F_{\sigma, \varphi(\alpha, t)}}{\left(G_{\zeta} * F_{\sigma, \varphi(\alpha, t)}\right)}\right)-\frac{\kappa}{2} \partial_{\phi}\left(G_{\zeta} * F_{\sigma, \varphi(\alpha, t)}\right)(\phi)\right] d \phi \\
= & 0,
\end{aligned}
$$

where we have integrated by parts. Using the exponential decay of $F$ at infinity, the boundary terms in the integration by parts vanish. The last equality is obtained as follows, using Eqs. (3.21) and (3.23):

$$
\begin{aligned}
G_{\zeta} * & \left(\frac{\left(\phi G_{\zeta} * F_{\sigma, \varphi(\alpha, t)}\right) F_{\sigma, \varphi(\alpha, t)}}{\left(G_{\zeta} * F_{\sigma, \varphi(\alpha, t)}\right)}\right)(\phi) \\
& =-\zeta^{2}\left(G_{\zeta} *\left(\frac{\left(\partial_{\phi}\left(G_{\zeta} * F_{\sigma, \varphi(\alpha, t)}\right)\right) F_{\sigma, \varphi(\alpha, t)}}{\left(G_{\zeta} * F_{\sigma, \varphi(\alpha, t)}\right)}\right)\right)(\phi) \\
& =-\zeta^{2}\left(G_{\zeta} *\left[\left(\partial_{\phi} \ln \left(G_{\zeta} * F_{\sigma, \varphi(\alpha, t)}\right)\right) F_{\sigma, \varphi(\alpha, t)}\right]\right)(\phi) \\
& =\zeta^{2} \frac{1}{\sigma^{2}+\zeta^{2}}\left(G_{\zeta} *(\phi-\varphi(\alpha, t)) F_{\sigma, \varphi(\alpha, t)}\right)(\phi) \\
& =-\zeta^{2} \frac{\sigma^{2}}{\sigma^{2}+\zeta^{2}}\left(G_{\zeta} * \partial_{\phi} F_{\sigma, \varphi(\alpha, t)}\right)(\phi) \\
& =-\frac{\kappa}{2} \partial_{\phi}\left(G_{\zeta} * F_{\sigma, \varphi(\alpha, t)}\right)(\phi) .
\end{aligned}
$$

In the last equality we used the value of $\sigma$ given in (3.17).

Finally, we can prove the following.

Theorem 4.1. Under the assumptions of Lemma 4.1, as $\varepsilon \rightarrow 0$, the solution $f^{\varepsilon}$ of (4.6) formally converges to a local thermodynamical equilibrium (4.9), i.e. $f^{\varepsilon}(\alpha, \varphi, t)=\rho(\alpha, t) F_{\sigma, \varphi(\alpha, t)}+\mathcal{O}\left(\varepsilon^{2}\right)$ in a suitable topology, where $\rho$ is constant in time and the density of opinion $\rho \varphi$ evolves according to the following diffusion equation in regions where $\rho>0$ :

(i) in the symmetric case,

$$
\frac{\partial}{\partial t}(\rho \varphi)=C_{s} \nabla_{\alpha} \cdot \rho^{2} \nabla_{\alpha} \varphi, \quad C_{s}:=\gamma D \frac{\zeta^{3}}{\left(2 \sigma_{s}^{2}+\zeta^{2}\right)^{3 / 2}},
$$

where $\sigma$ is given in Eq. (3.16); 
(ii) and in the non-symmetric case,

$$
\frac{\partial}{\partial t}(\rho \varphi)=\frac{C_{a}}{\rho} \nabla_{\alpha} \cdot \rho^{2} \nabla_{\alpha} \varphi, \quad C_{a}:=\gamma D \frac{\zeta^{2}}{\sigma_{a}^{2}+\zeta^{2}},
$$

where $\sigma$ is given by Eq. (3.17).

For the significance of this theorem we refer the reader to Sec. 2.

Proof of Theorem 4.1. From Lemma 4.1, we deduce that $f=f(\alpha, \phi, t)=$ $\rho(\alpha, t) F_{\sigma, \varphi(\alpha, t)(\varphi)}$. Notice that by assumption $G_{\zeta}$ is of the form (3.23). We split the proof between the symmetric case and the non-symmetric case.

Symmetric case. Successively multiplying Eq. (4.6) by 1 and $\varphi$ and using Lemma 4.2 we get

$$
\begin{aligned}
& \frac{\partial \rho^{\varepsilon}}{\partial t}(\alpha, t)=0, \\
& \frac{\partial}{\partial t}\left(\rho^{\varepsilon} \varphi^{\varepsilon}\right)(\alpha, t)+\gamma D \int_{\mathbb{R}^{2}}(\phi-\psi) G_{\zeta}(\phi-\psi) \Delta_{\alpha} f^{\varepsilon}(\alpha, \psi, t) \\
& \quad \times f^{\varepsilon}(\alpha, \phi, t) d \phi d \psi=0,
\end{aligned}
$$

where $\rho^{\varepsilon}$ and $\varphi^{\varepsilon}$ are defined by (4.10) with $f$ replaced by $f^{\varepsilon}$. Now, letting $\varepsilon \rightarrow 0$ and using (4.9), we get

$$
\begin{aligned}
& \frac{\partial \rho}{\partial t}(\alpha, t)=0, \\
& \frac{\partial}{\partial t}(\rho \varphi)(\alpha, t)+\gamma D \int_{\mathbb{R}^{2}}(\phi-\psi) G_{\zeta}(\phi-\psi) \Delta_{\alpha}\left(\rho(\alpha, t) F_{\sigma, \varphi(\alpha, t)}(\psi)\right) \\
& \quad \times \rho(\alpha, t) F_{\sigma, \varphi(\alpha, t)}(\phi) d \phi d \psi=0,
\end{aligned}
$$

We compute

$$
\nabla_{\alpha}\left(\rho(\alpha, t) F_{\sigma, \varphi(\alpha, t)}(\psi)\right)=F_{\sigma, \varphi(\alpha, t)}(\psi)\left[\nabla_{\alpha} \rho+\left(\frac{\psi-\varphi}{\sigma^{2}}\right) \rho \nabla_{\alpha} \varphi\right](\alpha, t),
$$

and

$$
\begin{aligned}
& \Delta_{\alpha}\left(\rho(\alpha, t) F_{\sigma, \varphi(\alpha, t)}(\psi)\right) \\
& =F_{\sigma, \varphi(\alpha, t)}(\psi)\left[\Delta_{\alpha} \rho-\frac{1}{\sigma^{2}} \rho\left|\nabla_{\alpha} \varphi\right|^{2}+\left(\frac{\psi-\varphi}{\sigma^{2}}\right)\left(\rho \Delta_{\alpha} \varphi+2 \nabla_{\alpha} \rho \cdot \nabla_{\alpha} \varphi\right)\right. \\
& \left.\quad+\left(\frac{\psi-\varphi}{\sigma^{2}}\right)^{2} \rho\left|\nabla_{\alpha} \varphi\right|^{2}\right](\alpha, t)
\end{aligned}
$$


Then, we have

$$
\begin{aligned}
-\frac{1}{\gamma D} \frac{\partial}{\partial t}(\rho \varphi)(\alpha, t)= & \rho\left[\Delta_{\alpha} \rho-\frac{1}{\sigma^{2}} \rho\left|\nabla_{\alpha} \varphi\right|^{2}\right](\alpha, t) \int_{\mathbb{R}^{2}}(\phi-\psi) G_{\zeta}(\phi-\psi) F_{\sigma, \varphi(\alpha, t)}(\psi) \\
& \times F_{\sigma, \varphi(\alpha, t)}(\phi) d \phi d \psi+\rho\left[\rho \Delta_{\alpha} \varphi+2 \nabla_{\alpha} \rho \cdot \nabla_{\alpha} \varphi\right](\alpha, t) \\
& \times \int_{\mathbb{R}^{2}} \frac{\psi-\varphi}{\sigma^{2}}(\phi-\psi) G_{\zeta}(\phi-\psi) F_{\sigma, \varphi(\alpha, t)}(\psi) F_{\sigma, \varphi(\alpha, t)}(\phi) d \phi d \psi \\
& +\rho^{2}\left|\nabla_{\alpha} \varphi\right|^{2}(\alpha, t) \int_{\mathbb{R}^{2}}\left(\frac{\psi-\varphi}{\sigma^{2}}\right)^{2}(\phi-\psi) G_{\zeta}(\phi-\psi) \\
& \times F_{\sigma, \varphi(\alpha, t)}(\psi) F_{\sigma, \varphi(\alpha, t)}(\phi) d \phi d \psi
\end{aligned}
$$

The first integral is identically zero by antisymmetry. The second and third integrals are denoted by $I_{2}$ and $I_{3}$. To compute them, we introduce the change of variables $\bar{\phi}=\phi-\varphi(\alpha, t), \bar{\psi}=\psi-\varphi(\alpha, t)$ and we get (omitting the bars for simplicity):

$$
\begin{aligned}
I_{2} & =\int_{\mathbb{R}^{2}} \frac{\psi}{\sigma^{2}}(\phi-\psi) G_{\zeta}(\phi-\psi) F_{\sigma, 0}(\psi) F_{\sigma, 0}(\phi) d \phi d \psi \\
I_{3} & =\int_{\mathbb{R}^{2}}\left(\frac{\psi}{\sigma^{2}}\right)^{2}(\phi-\psi) G_{\zeta}(\phi-\psi) F_{\sigma, 0}(\psi) F_{\sigma, 0}(\phi) d \phi d \psi=0 .
\end{aligned}
$$

The integral $I_{3}$ is identically 0 by antisymmetry. Now, we compute the integral $I_{2}$ using consecutively Eqs. (3.23), (3.22), (3.21) and (3.20):

$$
\begin{aligned}
I_{2} & =\int_{\mathbb{R}^{2}} \zeta^{2} \partial_{\phi} G_{\zeta}(\phi-\psi) \partial_{\psi} F_{\sigma, 0}(\psi) F_{\sigma, 0}(\phi) d \phi d \psi \\
& =\zeta^{2} \int_{\mathbb{R}}\left(\partial_{\phi} G_{\zeta} * \partial_{\phi} F_{\sigma, 0}\right)(\phi) F_{\sigma, 0}(\phi) d \phi \\
& =\zeta^{2} \int_{\mathbb{R}} \partial_{\phi}^{2}\left(G_{\zeta} * F_{\sigma, 0}\right)(\phi) F_{\sigma, 0}(\phi) d \phi \\
& =-\zeta^{2} \int_{\mathbb{R}} \partial_{\phi}\left(G_{\zeta} * F_{\sigma, 0}\right)(\phi) \partial_{\phi} F_{\sigma, 0}(\phi) d \phi \\
& =-\zeta^{2} \int_{\mathbb{R}} \sqrt{2 \pi} \zeta \partial_{\phi}\left(F \sqrt{\sigma^{2}+\zeta^{2}, 0}\right)(\phi) \partial_{\phi} F_{\sigma, 0}(\phi) d \phi \\
& =-\zeta^{3} \sqrt{2 \pi} \int_{\mathbb{R}} \frac{\phi^{2}}{\sigma^{2}\left(\sigma^{2}+\zeta^{2}\right) \sqrt{2 \pi\left(2 \sigma^{2}+\zeta^{2}\right)}} \sqrt{\frac{\sigma^{2}\left(\sigma^{2}+\zeta^{2}\right)}{2 \sigma^{2}+\zeta^{2}}, 0}(\phi) d \phi \\
& =-\frac{\zeta^{3}}{\left(2 \sigma^{2}+\zeta^{2}\right)^{3 / 2}} .
\end{aligned}
$$

Finally,

$$
\frac{1}{\gamma D} \frac{\partial}{\partial t}(\rho \varphi)(\alpha, t)=\frac{\zeta^{3}}{\left(2 \sigma^{2}+\zeta^{2}\right)^{3 / 2}} \rho\left[\rho \Delta_{\alpha} \varphi+2 \nabla_{\alpha} \rho \cdot \nabla_{\alpha} \varphi\right](\alpha, t),
$$

which gives the result for the symmetric case. 
Non-symmetric case. We conclude that $\rho=\rho(\alpha)$ is independent of time analogously as for the symmetric case. To compute the equation for $\varphi=\varphi(\alpha, t)$ we will use the collision-invariant for the linearized operator given in Lemma 4.3. We multiply Eq. (4.6) by

$$
\chi=\chi(\phi)=\int^{\phi}\left(G_{\zeta} * F_{\sigma, \varphi(\alpha, t)}\right)\left(\phi^{\prime}\right) d \phi^{\prime},
$$

and obtain

$$
\begin{aligned}
\int_{\mathbb{R}} \chi & (\phi)\left[\frac{\partial f_{\varepsilon}}{\partial t}-\gamma D \partial_{\phi}\left\{f_{\varepsilon}\left(\frac{\phi G_{\zeta} * \Delta_{\alpha} f_{\varepsilon}}{G_{\zeta} * f_{\varepsilon}}-\frac{\left(\phi G_{\zeta} * f_{\varepsilon}\right)\left(G_{\zeta} * \Delta_{\alpha} f_{\varepsilon}\right)}{\left(G_{\zeta} * f_{\varepsilon}\right)^{2}}\right)\right\}\right](\phi) d \phi \\
& =\frac{1}{\varepsilon^{2}} \int_{\mathbb{R}} Q\left(f_{\varepsilon}\right)(\phi) \chi(\phi) d \phi .
\end{aligned}
$$

Now writing that $f_{\varepsilon}=\rho(\alpha, t) F_{\sigma, \varphi(\alpha, t)}+\varepsilon^{2} f_{1}+o\left(\varepsilon^{2}\right)$ (by assumption), we have that $Q\left(f_{\varepsilon}\right)=\varepsilon^{2} \operatorname{Lin}_{Q}\left(f_{1}\right)+o\left(\varepsilon^{2}\right)$, where $\operatorname{Lin}_{Q}$ is given in (4.11). Consequently, by Lemma 4.3, it holds that

$$
\frac{1}{\varepsilon^{2}} \int Q\left(f_{\varepsilon}\right)(\phi) \chi(\phi) d \phi \underset{\varepsilon \rightarrow+0}{\longrightarrow} 0
$$

We are left with computing the left-hand side of Eq. (4.19). Letting $\varepsilon \rightarrow 0$, keeping the highest order in $\varepsilon$ and integrating by parts, we obtain

$$
\begin{aligned}
0= & \int_{\mathbb{R}} \chi(\phi) \frac{\partial f}{\partial t} d \phi+\gamma D \int_{\mathbb{R}}\left(G_{\zeta} * F_{\sigma, \varphi(\alpha, t)}\right) f \\
& \times\left[\frac{\phi G_{\zeta} * \Delta_{\alpha} f}{G_{\zeta} * f}-\frac{\left(\phi G_{\zeta} * f\right)\left(G_{\zeta} * \Delta_{\alpha} f\right)}{\left(G_{\zeta} * f\right)^{2}}\right](\phi) d \phi \\
:= & I_{1}+I_{2}+I_{3},
\end{aligned}
$$

with $f=\rho(\alpha) F_{\sigma, \varphi(\alpha, t)}$. Next we compute each one of the terms. For $I_{1}$ we obtain, using again Eqs. (3.20)-(3.23),

$$
\begin{aligned}
I_{1} & =\int_{\mathbb{R}} \frac{\partial f}{\partial t}(\phi) \chi(\phi) d \phi \\
& =\rho(\alpha) \int_{\mathbb{R}} \frac{\partial F_{\sigma, \varphi(\alpha, t)}}{\partial t}(\phi) \chi(\phi) d \phi \\
& =\frac{\partial \varphi}{\partial t}(\alpha, t) \rho(\alpha) \int_{\mathbb{R}} \partial_{\phi} F_{\sigma, \varphi(\alpha, t)}(\phi) \chi(\phi) d \phi \\
& =\frac{\partial \varphi}{\partial t}(\alpha, t) \rho(\alpha) \int_{\mathbb{R}} F_{\sigma, \varphi(\alpha, t)}(\phi)\left(G_{\zeta} * F_{\sigma, \varphi(\alpha, t)}\right)(\phi) d \phi \\
& =\sqrt{2 \pi} \zeta \frac{\partial \varphi}{\partial t}(\alpha, t) \rho(\alpha) \int_{\mathbb{R}} F_{\sigma, \varphi(\alpha, t)}(\phi) F_{\sqrt{\sigma^{2}+\zeta^{2}}, \varphi(\alpha, t)}(\phi) d \phi
\end{aligned}
$$




$$
\begin{aligned}
& =\sqrt{2 \pi} \zeta \frac{\partial \varphi}{\partial t}(\alpha, t) \rho(\alpha) \frac{1}{\sqrt{2 \pi\left(2 \sigma^{2}+\zeta^{2}\right)}} \int_{\mathbb{R}} F \sqrt{\frac{\sigma^{2}\left(\sigma^{2}+\zeta^{2}\right)}{2 \sigma^{2}+\zeta^{2}}, \varphi(\alpha, t)}(\phi) d \phi \\
& =\sqrt{2 \pi} \zeta \frac{\partial \varphi}{\partial t}(\alpha, t) \rho(\alpha) \frac{1}{\sqrt{2 \pi\left(2 \sigma^{2}+\zeta^{2}\right)}} .
\end{aligned}
$$

The integral

$$
\begin{aligned}
I_{2} & =\gamma D \int_{\mathbb{R}} F_{\sigma, \varphi(\alpha, t)}(\phi)\left(\phi G_{\zeta} * \Delta_{\alpha} f\right)(\phi) d \phi \\
& =-\gamma D \frac{\zeta^{3}}{\left(2 \sigma^{2}+\zeta^{2}\right)^{3 / 2}}\left[\rho \Delta_{\alpha} \varphi+2 \nabla_{\alpha} \rho \cdot \nabla_{\alpha} \varphi\right]
\end{aligned}
$$

was already computed in the symmetric case (observe that the factor $\rho$ is not present here), so we are left with computing

$$
I_{3}=-\gamma D \int_{\mathbb{R}} F_{\sigma, \varphi(\alpha, t)}(\phi)\left(\frac{\phi G_{\zeta} * F_{\sigma, \varphi(\alpha, t)}\left(G_{\zeta} * \Delta_{\alpha}\left(\rho(\alpha) F_{\sigma, \varphi(\alpha, t)}\right)\right)}{G_{\zeta} * F_{\sigma, \varphi(\alpha, t)}}\right)(\phi) d \phi .
$$

Using the computation for $\Delta_{\alpha}\left(\rho F_{\sigma, \varphi(\alpha, t)}\right)$ in Eq. (4.18), we can compute analogously as for the symmetric case. One can check that the only term that does not cancel by an antisymmetry argument (as done previously for the integrals $I_{1}$ and $I_{3}$ in the symmetric case) is

$$
\begin{aligned}
& I_{3}=\left[\rho \Delta_{\alpha} \varphi+2 \nabla_{\alpha} \rho \cdot \nabla_{\alpha} \varphi\right] \gamma D \int_{\mathbb{R}} F_{\sigma, \varphi(\alpha, t)}(\phi) \frac{\left(\phi G_{\zeta} * f\right)\left(G_{\zeta} * \partial_{\phi} F_{\sigma, \varphi(\alpha, t)}\right)}{\left(G_{\zeta} * f\right)}(\phi) d \phi \\
& =-\zeta^{2}\left[\rho \Delta_{\alpha} \varphi+2 \nabla_{\alpha} \rho \cdot \nabla_{\alpha} \varphi\right] \gamma D \\
& \times \int_{\mathbb{R}} F_{\sigma, \varphi(\alpha, t)}(\phi) \frac{\partial_{\phi}\left(G_{\zeta} * F_{\sigma, \varphi(\alpha, t)}\right) \partial_{\phi}\left(G_{\zeta} * F_{\sigma, \varphi(\alpha, t)}\right)}{\left(G_{\zeta} * F_{\sigma, \varphi(\alpha, t)}\right)}(\phi) d \phi \\
& =\zeta^{2}\left[\rho \Delta_{\alpha} \varphi+2 \nabla_{\alpha} \rho \cdot \nabla_{\alpha} \varphi\right] \gamma D \\
& \times \int_{\mathbb{R}} F_{\sigma, \varphi(\alpha, t)}(\phi) \partial_{\phi}\left(G_{\zeta} * F_{\sigma, \varphi(\alpha, t)}(\phi)\right) \frac{(\phi-\varphi)}{\sigma^{2}+\zeta^{2}}(\phi) d \phi \\
& =-\sqrt{2 \pi} \zeta^{3}\left[\rho \Delta_{\alpha} \varphi+2 \nabla_{\alpha} \rho \cdot \nabla_{\alpha} \varphi\right] \gamma D
\end{aligned}
$$

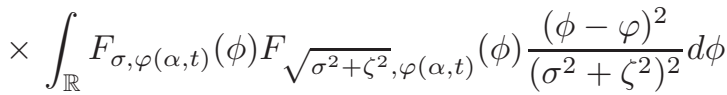

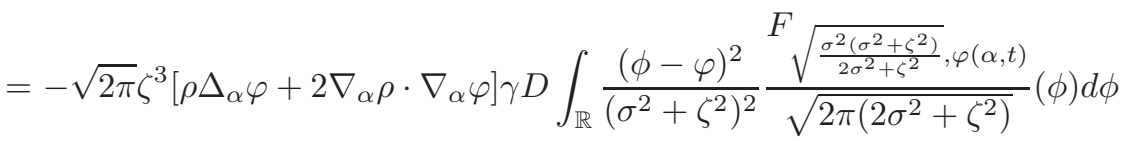

$$
\begin{aligned}
& =-\sqrt{2 \pi} \zeta^{3}\left[\rho \Delta_{\alpha} \varphi+2 \nabla_{\alpha} \rho \cdot \nabla_{\alpha} \varphi\right] \frac{\gamma D \sigma^{2}}{\left(\sigma^{2}+\zeta^{2}\right) \sqrt{2 \pi\left(2 \sigma^{2}+\zeta^{2}\right)}\left(2 \sigma^{2}+\zeta^{2}\right)} .
\end{aligned}
$$

We conclude

$$
\rho \frac{\partial \varphi}{\partial t}=\gamma D \frac{\zeta^{2}}{\sigma^{2}+\zeta^{2}}\left[\rho \Delta_{\alpha} \varphi+2 \nabla_{\alpha} \rho \cdot \nabla_{\alpha} \varphi\right]
$$




\section{Conclusions}

In this work, we have studied the long-time dynamics of an opinion model in the limit of spatially localized interactions under the assumption of low consensus. The considered model is borrowed from Ref. 10 where it is used to model voter's intentions. We have investigated the dynamics under two different interaction rates: one given in Ref. 10 (symmetric binary interactions) and the other inspired from Refs. 29 and 30 (non-symmetric binary interactions). In particular, we show that the density of opinion $\rho \varphi$ is a conserved quantity for the symmetric case but not for the nonsymmetric case; this is a direct translation of the conservative (or non-conservative) properties of their respective kinetic equations. Moreover, in Corollary 2.1 we give criterion on the spatial density $\rho$ to decide for which one of the two rates consensus is reached faster.

The interaction rates considered come with a modeling choice: the symmetric rate in Ref. 10 assumes, for instance, that any two pairs of individuals have the same influence on each other; this is not the case for the non-symmetric rate, which assumes that individuals in a large group are more influential than solitary ones. Other modeling assumptions can be considered, for example, we could assume transport of individuals between different spatial regions. In this manner, one could assume that individuals move toward places where larger clusters are formed (migration from the countryside to the cities) or a rule where individuals move to places where others think alike. As we can see, a variety of possible modeling assumptions can modify these opinion models in various directions. The validity of the model considered will depend on the actual situation that we wish to describe. In any case, the derivation of the macroscopic equations for these models leads, first, to the long-time dynamics, and second, to understanding the relevance of a particular modeling choice and contrast it with other alternative choices. Here, we have illustrated this idea by comparing the conservative properties and the influence on the speed toward consensus for two different interaction rates.

Deriving macroscopic equations for these models has many benefits: it gives access to the long-time dynamics in both a qualitative way (by analyzing the dependence of the diffusion constants on the model parameters) and in a quantitative way (by enabling numerical simulations for a large number of agents and over a long time without suffering from the curse of complexity). It also allows us to explore the relevance of particular modeling choices by contrasting them with alternate choices (illustrated here by the striking differences between two different interaction models in their conservation properties and speed toward consensus). For the non-symmetric case, we were led to develop a theory beyond the state-of-the-art of kinetic theory which is mostly restricted to the conservative case (see however Ref. 19 for another instance of treatment of non-conservative interactions). Being able to treat non-conservative interactions is key toward the development of kinetic theory for social interactions as such interactions cannot be associated with a conserved quantity in general. 


\section{Acknowledgments}

P.D. acknowledges support from the Royal Society and the Wolfson Foundation through a Royal Society Wolfson Research Merit Award; the British "Engineering and Physical Research Council" under Grant Ref.: EP/M006883/1; the National Science Foundation under NSF Grant RNMS11-07444 (KI-Net). P.D. is on leave from CNRS, Institut de Mathématiques de Toulouse, France. P.D. is grateful to J.-P. Bouchaud for suggesting the problem and for stimulating discussions. J.G.L. was partially supported by KI-Net NSF RNMS Grant No. 1107291 and NSF Grant DMS 1514826. S.M.A. was supported by the British "Engineering and Physical Research Council" under Grant Ref.: EP/M006883/1. T.T. gratefully acknowledges the hospitality of the Department of Mathematics at Imperial College London, where this research was conducted.

\section{Data statement}

No new data was generated in the course of this research.

\section{References}

1. I. Aoki, A simulation study on the schooling mechanism in fish, Bull. Jpn. Soc. Sci. Fish. 48 (1982) 1081-1088.

2. R. Axelrod, The dissemination of culture. A model with local convergence and global polarization, J. Conflict Resolut. 41 (1997) 203-206.

3. N. Bellomo, A. Bellouquid and D. Knopoff, From the microscale to collective crowd dynamics, Multiscale Model. Simulat. 11 (2013) 943-963.

4. E. Ben-Naim, Opinion dynamics: Rise and fall of political parties, Europhys. Lett. 69 (2005) 671.

5. E. Ben-Naim, P. L. Krapivsky and S. Redner, Bifurcations and patterns in compromise processes, Physica D 183 (2003) 190-204.

6. E. Ben-Naim and S. Redner, Dynamics of social diversity, J. Stat. Mech. Theory Exp. 11 (2005) L11002.

7. H. Berestycki, J.-M. Roquejoffre and L. Rossi, Travelling waves, spreading and extinction for Fisher-KPP propagation driven by a line with fast diffusion, Nonlinear Anal. 137 (2016) 171-189.

8. C. Borghesi and J.-P. Bouchaud, Spatial correlations in vote statistics: A diffusive field model for decision-making, Eur. Phys. J. B 75 (2010) 395-404.

9. C. Borghesi, J.-C. Raynal and J.-P. Bouchaud, Election turnout statistics in many countries: Similarities, differences, and a diffusive field model for decision-making, PLoS ONE 7 (2012) e36289.

10. J.-P. Bouchaud, C. Borghesi and P. Jensen, On the emergence of an "intention field" for socially cohesive agents, J. Stat. Mech. Theory Exp. 3 (2014) P03010.

11. E. Carlen, M. C. Carvalho, P. Degond and B. Wennberg, A Boltzmann model for rod alignment and schooling fish, Nonlinearity 28 (2015) 1783-1803.

12. C. Castellano, S. Fortunato and V. Loreto, Statistical physics of social dynamics, Rev. Mod. Phys. 81 (2009) 591-646.

13. C. Cercignani, R. Illner and M. Pulvirenti, The Mathematical Theory of Dilute Gases (Springer Science \& Business Media, 2013). 
14. S. Cordier, L. Pareschi and G. Toscani, On a kinetic model for a simple market economy, J. Stat. Phys. 120 (2005) 253-277.

15. G. Deffuant, D. Neau, F. Amblard and G. Weisbuch, Mixing beliefs among interacting agents, Adv. Complex Syst. 3 (2001) 87-98.

16. P. Degond, S. Génieys and A. Jüngel, Symmetrization and entropy inequality for general diffusion equations, C. R. Math. Acad. Sci. Paris 325 (1997) 963-968.

17. P. Degond, J.-G. Liu and C. Ringhofer, Evolution of wealth in a nonconservative economy driven by local Nash equilibria, Philos. Trans. A 372 (2014) 20130394.

18. P. Degond and B. Lucquin-Desreux, The Fokker-Planck asymptotics of the Boltzmann collision operator in the Coulomb case, Math. Models Methods Appl. Sci. 2 (1992) $167-182$.

19. P. Degond and S. Motsch, Continuum limit of self-driven particles with orientation interaction, Math. Models Methods Appl. Sci. 18 (2008) 1193-1215.

20. P. Degond and S. Motsch, Collective dynamics and self-organization: Some challenges and an example, ESAIM Proc. Surveys 45 (2014) 1-7.

21. B. Düring and M.-T. Wolfram, Opinion dynamics: Inhomogeneous Boltzmann-type equations modelling opinion leadership and political segregation, Proc. R. Soc. A 471 (2015) 20150345.

22. J. Evers and T. Kolokolnikov, Metastable states for an aggregation model with noise, arXiv: 1604.00735 .

23. S. Galam, Minority opinion spreading in random geometry, Eur. Phys. J. B 25 (2002) 403-406.

24. S. Galam, Y. Gefen and Y. Shapir, Sociophysics: A new approach of sociological collective behavior, J. Math. Sociol. 9 (1982) 1-13.

25. E. Geigant and M. Stoll, Stability of peak solutions of a nonlinear transport equation on the circle, arXiv: 1108.5462 .

26. D. Helbing, Traffic and related self-driven many-particle systems, Rev. Mod. Phys. $\mathbf{7 3}$ (2001) 1067.

27. D. Helbing, A. Johansson and H. Z. Al-Abideen, Dynamics of crowd disasters: An empirical study, Phys. Rev. E 75 (2007) 046109.

28. M. Lachowicz, H. Leszczynski and M. Parisot, A simple kinetic equation of swarm formation: Blow-up and global existence, Appl. Math. Lett. 57 (2016) 104-107.

29. S. Motsch and E. Tadmor, A new model for self-organized dynamics and its flocking behavior, J. Stat. Phys. 144 (2011) 923-947.

30. S. Motsch and E. Tadmor, Heterophilious dynamics enhances consensus, SIAM Rev. 4 (2014) 577-621.

31. T. Schelling, Dynamic models of segregation, J. Math. Soc. 1 (1971) 143-186.

32. F. Slanina and H. Lavicka, Analytical results for the Sznajd model of opinion formation, Eur. Phys. J. B 35 (2003) 279-288.

33. K. Sznajd-Weron, Sznajd model and its applications, Acta Phys. Polon. B 36 (2005) $2537-2547$.

34. G. Toscani, Kinetic models of opinion formation, Commun. Math. Sci. 4 (2006) 481496.

35. W. Weidlich, The statistical description of polarization phenomena in society, British J. Math. Stat. Psychol. 24 (1971) 251-266.

36. S. Wichmann, The emerging field of language dynamics, arXiv: 0801.1415W.

37. M. Yamao, H. Naoki and S. Ishii, Multi-cellular logistics of collective cell migration, PLoS ONE 6 (2011) e27950. 